\title{
LAS OSCILACIONES CLIMÁTICAS EN LA ATMOSFERA URBANA DE LA CIUDAD DE NATAL, BRASIL ATRAVÉS DE LAS SERIES LARGAS DE TEMPERATURAS DEL AIRE
}

\author{
M. J. O. Alexandre' ; F. F. García ${ }^{2}$; E. Nunes ${ }^{3}$ \\ ${ }^{1}$ Instituto Federal do Rio Grande do Norte \\ ${ }^{2}$ Universidad Autónoma de Madrid \\ ${ }^{3}$ Universidade Federal do Rio Grande do Norte \\ E-mail: malco.alexandre@ifrn.edu.br ${ }^{1}$
}

Artigo submetido em outubro/2013 e aceito em outubro/2013

\section{RESUMEN}

Este estudio tiene como objetivo el análisis de la isla de calor urbana (ICU) y de la isla de frescor urbana (IFU) en la ciudad de Natal (Brasil), mediante la comparación de las series largas de temperatura del aire máximas y mínimas diarias en dos estaciones meteorológicas convencionales representativas una en la área urbana y la otra en el entorno, durante el quinquenio 1995-2010. Los resultados muestran la presencia de de oscilaciones térmicas a partir del contraste entre las temperaturas del área urbana y su entorno y la caracterización de las islas de calor y frescor urbano en la ciudad de Natal.

PALABRAS CLAVE: Isla de calor de la atmosfera urbana (ICU), Isla de frescor de la atmosfera urbana (IFU), Ciudad de Natal.

\section{WEATHER OSCILLATIONS IN THE URBAN ATMOSPHERE OF THE CITY OF NATAL, BRAZIL THROUGH THE LONG SERIES AIR TEMPERATURE}

\begin{abstract}
This study aims at analyzing the urban heat island (UHI) and urban cool island ( $\mathrm{UCl}$ ) in the city of Natal (Brazil), by comparing the long series of air temperature maximum and minimum daily into two weather stations conventional representative, one in the urban area and the other in the rural area, during the fifteen years, from
\end{abstract}

1995 to 2010. The result shows the presence of thermal fluctuations from the temperature contrast between the urban area and its surroundings area and characterization of urban heat islands and urban cool island in the city of Natal.

KEYWORDS: Urban Heat Island (UHI), Urban Cool Island (UCI), City of Natal. 


\section{LAS OSCILACIONES CLIMÁTICAS EN LA ATMOSFERA URBANA DE LA CIUDAD DE NATAL, BRASIL ATRAVÉS DE LAS SERIES LARGAS DE TEMPERATURAS DEL AIRE}

\section{INTRODUCCIÓN}

El siglo XX fue el siglo del ambientalismo y de la urbanización. Del ambientalismos principalmente con El marco de 1972 enla primera Conferencia de lasNaciones Unidas sobre Medio Ambiente donde los temas relacionados con el medio ambiente tomaron dimensiones mayorestrayendo para la agenda política internacional la dimensión ambiental como factor condicionante para el crecimientoeconómico. De la urbanización con el marco en el año 2000 cuando la población mundial supera el campo en más de $50 \%$ en las ciudades. Además, entre los principales temas ambientales tratados a nivel mundial el cambio climático ha puesto de relieve (Fernández, 2010).

Stewart y Oke (2012) destacan entre los principales problemas ambientales del século XXI el crecimiento demográfico y el cambio climático e implica el tema de las islas de calor urbano como un problema importante y grave. De esto explicase, desde uno de los estudios pioneros de clima urbano la monografia The climate of London de Tony Chandler 1965 el notable crecimiento principalmente a partir de las ultimas décadas del numero de estudios sobre las islas de calor urbano en todo el mundo, incluyendo las grandes metropoles hasta las pequenas ciudades.

Hay pocas dudas en relación al cambio climático, pero hay dudas sobre su dimensión y su naturaleza natural y/o antropica. Pero, en escalas pequeñas de ámbito urbano, no hay dudas de que el hombre está cambiando el clima. Admitiendo que el hombre cambia el clima en escalas pequeñas ¿por qué ocurre en las ciudades? El pensamiento Miltoniano afirma que las ciudades estan como un punto de intercepción entre la verticalidad y la horizontalidad, en cuanto las horizontalidades están sobre todo, los fijos, el lugar, la ciudad propiamente dita y el locus de la producción y en la verticalidad están sobre todo los otros momentos de la producción, la circulación, la distribución y el consumo (Santos, 1999). Corrêa (1989) define el espacio urbano como un complexo conjunto de usos diferenciados de la tierra, tales usos definen el centro de la ciudad, áreas industriales, áreas residenciales y áreas comerciales.

Conforme los conceptos anteriores, en la ciudad están las trasformaciones impuestas por la producción en un determinado espacio, entre otras transformaciones estas pueden ser, las estructuras urbanas, avenidas, calles, plazas, edificaciones etc. eso todo por su parte influye artificialmente en las oscilaciones atmosféricas locales.

Así pues, conviene resaltar que son las ciudades un medio modificador de las condiciones atmosféricas de su espacio geográfico (Fernández García, F....Gil y Olcina, 2006). Donde el aire en el dosel urbano suele ser más cálido que el medio rural o los alrededores circundantes de la ciudad o el campo y esto por su vez forma las islas térmicas, las isla de calor urbano (urban heat island). El efecto de la ICU es probablemente la más clara muestra documental de la modificación inadvertida del clima (Oke, 1987).

La presencia de las islas de calor de la atmósfera urbana (ICU) y su opuesto la isla de frescor de la atmosfera urbana (IFU), ya es un conocido problema climático y ambiental en 
muchas ciudades del mundo, principalmente debido al fenómeno del fuerte crecimiento urbano. Las ciudades son aglomeraciones que en sentido más amplio forman manchas donde producen una distribución espacial diferenciada de la temperatura en la área urbana, que es, el efecto de la isla de calor (Olivier, 2005).

El fenómeno de la isla de calor urbana no es un problema exclusivo de las ciudades templadas de media y alta latitudes y altitudes. Ocurre tanto en estas, como también en las ciudades tropicales o de bajas latitudes (Barry y Chorley, 2003).

La presencia y tendencia tropicales de las islas de calor son muy parecidas con las ciudades templadas pero suelen ser más débiles con distribuciones diferentes para las temperaturas. Hay que destacar que, en algunas ciudades tropicales y costeras el aumento de intensidad de la temperatura vespertina puede producir un efecto térmico urbano contrario formando una isla de frescor (Barry y Chorley, 2003). Otra situación, como es de esperar, es que, en las ciudades tropicales los efectos de una fuerte ICU puede carrear serios problemas ambientales, económicos y sociales.

Así, el objetivo principal de este estudio es analizar la temperatura de la atmósfera urbana en la ciudad de Natal a través del levantamiento de los datos de las series largas de las temperaturas del aire con base en dos estaciones meteorológicas, una en la ciudad de Natal y la otra en el aeropuerto, permitiendo hacer un estudio comparativo para diagnosticar las posibles variaciones de las temperaturas que suelen representar la formación de la isla de calor o isla de frescor urbana.

Indudablemente la mejora de calidad ambiental en las ciudades es un factor muy importante y entre esto está la cuestión climática que, desgraciadamente aún no es o no está muy relevante en las principales políticas ambientales municipales principalmente en los países emergentes, como Brasil.

\section{BREVE RESUMEN DE LOS ESTUDIOS DE CLIMA URBANO EN BRASIL}

Los estudios de clima urbano en Brasil comenzó a partir de la década de 1970 y en especial con la contribución de la Teoria Sistema Clima Urbano de Monteiro (1984), fue sin duda, el marco que ha canalizó muchos de los estudios posteriores (Monteiro y Mendonça, 2003).

Hay una gran diversidad de estudios referentes al clima urbano en Brasil aplicados al estudio de las islas de calor y diversas posibilidades metodológicas combinadas tales como, estúdios comparativos de las temperaturas entre estaciones meteorológicas urbanas y rurales, estudios utilizando una red de estaciones meteorológicas disperso en todo tejido urbano, el método de los transectos móbiles y el uso y difusión de las imágenes satelitales (FIALHO, 2012).

Zavattini (2004) hizo una importante avaliación de 108 estudios científicos entre tesis y disertaciones de los climas en Brasil entre los años de 1971 hasta 2000 desarrolados y defendidos en algunas universidades brasileñas. De estos un número considerable de 40 estudios refierese a la climatologia urbana. 
Assis y Pereira (2005, apud Assis, 2006) hicieron un estudio de la producción brasileña en el clima urbano, conforto ambiental y eficiencia energética en los ultimos diez años (1995-2005) en un estudio hecho para las Centrales Eléctricas Brasileñas (Eletrobras). En total fueron más de 170 trabajos en el tema, entre libros, capitulos de libros, tesis, disertaciones y artículos científicos. De ese total, alrededor del 77\% fueron publicados por grupos de investigacion de la arquitectura o ingeniaria, mientras $23 \%$ de la geografia y meteorología involucrados en 23 instituiciones brasileñas de investigacion. La variable más estudiada fue la temperatura del aire, pero solamente $10 \%$ de los temas estudiados refierense a las islas de calor, además de $47 \%$ de la metodologia utilizada fue a través de estaciones meteorológicas fijas o movil.

A continuación se presenta un breve resumen de algunos estudios de clima urbano en Brasil que describen las islas de calor y de frescor o de la diferencia de temperaturas entre las zonas urbanas y rurales, se eligieron cinco ciudades, algunas con similares características climáticas y urbanas en relación a Natal.

Brandão (2003) ha desarrolló un estúdio del clima urbano en la ciudad de Rio de Janeiro, con la metodologia principalmente de los transectos móbiles y de estaciones meteorológicas fijas. En los resultados se encontraron varias islas de calor y de frescor con intensidades y temporalidades distintas. Los sistemas sinópticos predominantes fueron con el Anticiclone Polar muy instable y no favorable a la formacion de islas de calor pero en la presencia del Anticiclone Subtropical con cielos abiertos y despejados muy favorable a la formacion de islas de calor. Estas en Rio de Janeiro tienem máxima intensidad a las $15 \mathrm{~h}$ y $21 \mathrm{~h}$ en periodo de invierno y otoño, las intensidades de moderada a fuerte $\left(4{ }^{\circ} \mathrm{C}\right.$ a $5 \circ \mathrm{C}$ ) y las islas de frescor presentes muy a menudo en las áereas verdes como en el parque del Jardin Botanico. Además las islas de calor tienden a desvanecerse en el periodo nocturno con la actuacción del paso de frente frontal, con instabilidad, lluvia, alta humedad y nubes.

Moreira y Galvíncio (2007) en su estudio sobre la distribución geográfica de las temperaturas de la superficie en la ciudad de Recife (que se encuentra a $286 \mathrm{~km}$ de Natal) con imágenes de Landsat, nos reveló que la variación espacial de las temperaruras de superficie presenta dos áreas distintas, una al norte y la otra al sur de la ciudad, añadimos que Recife tiene la particularidad de tener su área entrecortada por rios donde el centro de la ciudad es formado por islas. Por eso, las áreas de temperaturas mas bajas se asocian con áreas de vegetacion (como los manglares) y tambien de los cuerpos hidricos. De acuerdo con los resultados, la ciudad presenta máximas islas de calor de intensidad fuerte con amplitud de 5으 $\mathrm{C}$ la zona sur cerca del aeropuerto y en el barrio de Boa Viagem.

Moura, Zanella y Sales (2008) analizaron las islas térmicas en la ciudad de Fortaleza CE en dos experimentos a través de doce puntos de observaciones en la área urbana. El primero experimento fue en la temporada de lluvias sobre acción del sistema sinóptico de la Zona de Convergencia Intertropical (ZCIT) donde las islas de calor son frecuentes por la mañana y tarde con valores hasta 4,7 으. En el segundo experimento en la época de seca sobre condiciones sinópticas de estabilidad atmosférica con actuación de la Masa Tropical Atlántica (mTa) las islas de calor estuvieron presentes en todos momentos del día con niveles hasta 5으. En los dos experimentos las islas de calor son menos frecuentes durante la noche. Como resultado Fortaleza 
tiene las islas de calor durante el día, en el centro de la ciudad y en el sector suroeste y las dos áreas con bajos niveles de vegetación.

Araújo y Rangel (2012) hicieron un estúdio del crescimiento urbano y las variaciones térmicas en la ciudad de São Luis MA. El municipio de São Luis ocupa la mayor parte de una isla, con clima ecuatorial caliente y húmedo, la temperatura media del aire de $28^{\circ} \mathrm{C}$, con vientos dominantes de NE y velocidad media de $6 \mathrm{~m} / \mathrm{s}$. En este trabajo se analizaron dos imágenes termales del año 1992 y 2010 . El resultado señala que hubo el aumento significativo de la temperatura superficial en el área urbana entre 1992 alrededor de 35 으 a 37으 en 2010 . El estudio también muestra un predominio de temperaturas superficiales más altas en la zona de mayor densidad urbana y con la cubierta vegetal reducida y la formación de islas de calor en diferentes partes de la zona urbana.

Saraiva, Vale y Zanella (2012) en ese estudio evaluaron las diferencias de las temperaturas del aire entre el área urbana y la rural de la ciudad de Mossoró. Municipio que se inserta en la región semiárida del nordeste de Brasil con características más destacadas de las altas temperaturas, el aire seco y la escasez de lluvia. El análisis fue hecho a través de seis puntos de observación ( 5 en la zona urbana y 1 en la zona rural) empezando en el día 05/04/2012 hasta el día 06/05/2012 durante las 24 horas del día y en un episodio de otoño. Los resultados indicaron una isla muy fuerte hasta $7,9 \circ \mathrm{C}$ al mediodía en el barrio de Santo Antonio cerca al centro de la ciudad, un barrio con alta densidad urbana, calles pavimentadas y poca vegetación. El estudio muestra islas de calor diurnas y las amplitudes térmicas más bajas se encuentran entre las 23 horas hasta las 2 horas (periodo nocturno).

Los estudios existentes en la literatura internacional muestran los resultados para las ciudades de clima templado, con una clara diferencia entre las máximas y mínimas y la formación de laS típicaS islas de calor nocturnas (Oke, 1995. p. 299).

Barry y Chorley (2003) evaluaron que las tendencias tropicales de las islas de calor suelen ser más débiles que en las ciudades templadas además, las tasas de enfriamiento y calentamiento en las zonas urbanas son más lentas que en las zonas rurales y esto hace que el principal efecto nocturno de las islas se manifiesten más tarde que las ciudades templadas, retrasando para el periodo del amanecer y diurnas. Una conclusión preliminar sugiere que de acuerdo con los estudios anteriores presentados, las islas de calor en ciudades de baja latitud y de clima tropical predominan en el periodo diurno.

Mendonça (2003) señala que las islas de calor nocturno en las ciudades templadas corroboron y son benéficas para la sociedad, pero en ciudades tropicales con mayores niveles de calor urbano vespertinos y nocturnos, lo más importante es la presencia de islas de frescor urbana.

\section{EL CLIMA URBANO DE NATAL: ÁREA DE ESTUDIO}

La ciudad de Natal es la capital del estado del estado del Rio Grande del Norte ubicada en el Noreste de Brasil con coordenadas centrales de 5047'42"(S) y 3512'34"(O) con un área de aproximadamente de 168,53 km² (Natal, 2012), población estimada de 803.739 habitantes y una 
densidad poblacional de $4.182 \mathrm{hab} / \mathrm{km}$ (Figura 1). La ciudad de Natal junto a otros 10 municipios circundantes forman la Región Metropolitana de Natal (RMN), también conocida como Gran Natal y con una población total estimada de 1.322.985 (IBGE, 2012). Las principales actividades económicas del municipio están divididas entre $82 \%$ servicios, $17 \%$ industria y casi no hay agropecuaria $0,2 \%$, por lo tanto, la área urbana ocupa toda la extensión de su área territorial (IBGE, 2012).
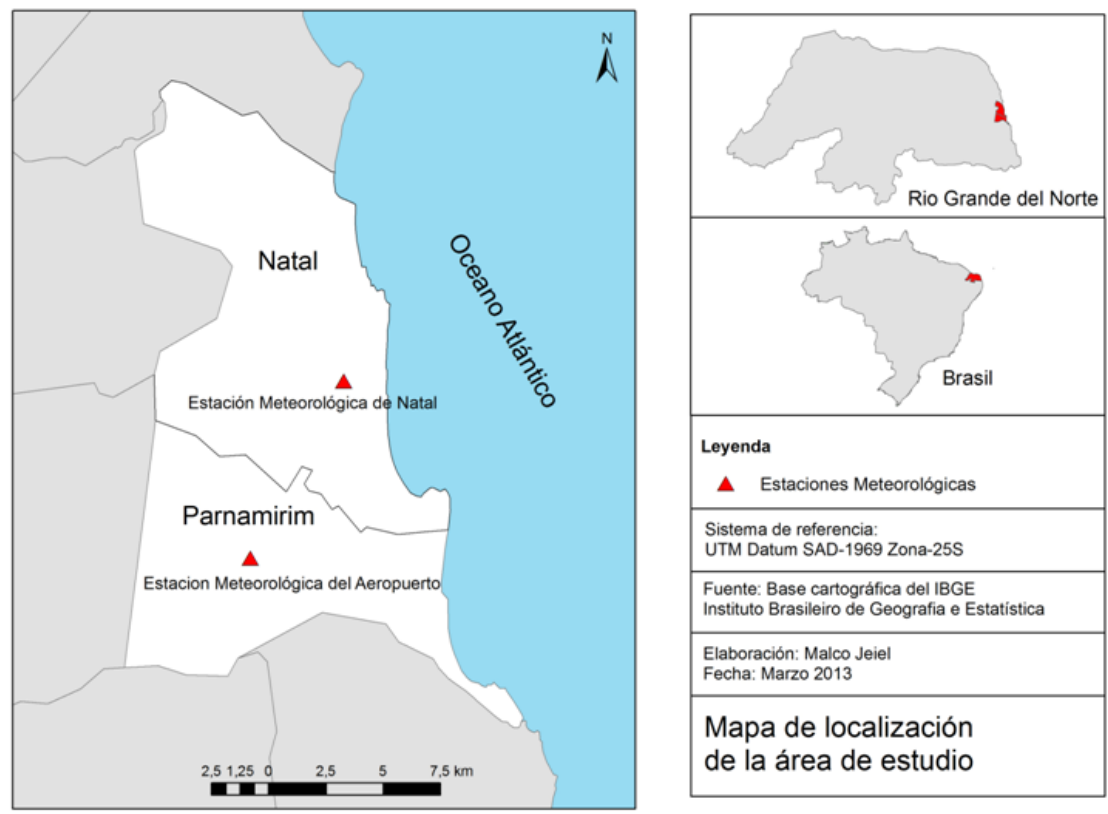

Figura 1: Mapa de localización de la área de estudio. Fuente base cartográfica del IBGE.

El clima de la ciudad de Natal está dentro de la Zona Climática Tropical y la región presenta dos estaciones distintas con mayor predominancia de lluvias en otoño y invierno en los meses de abril, mayo, junio y julio y seco en los meses de octubre y noviembre (primavera) predominantemente en diciembre, enero y febrero (verano). Las situaciones sinópticas predominantes están a menudo muy asociadas, entre otros, al desplazamiento de la Zona de Convergencia Intertropical (ZCIT) y de los Sistemas Frontales y de los vientos Alisios del sureste procedentes del océano Atlántico Sur (Baldicero Molion; Oliveira Bernardo, 2002).

Debido a sus características de tropicalidad las estaciones climáticas en Natal están condicionadas a las lluvias (Ayoade, 2003). Así, el final del otoño y el invierno son las estaciones de las lluvias donde hay un superávit de $1.040 \mathrm{~mm}$ de agua que se distribuye entre febrero y agosto, con una humedad relativa promedio de $77 \%$ y temperatura anual con un promedio de $26,6^{\circ} \mathrm{C}$ (RN, SEPLAN, IDEMA, 2010, p.25). La Figura 2 presenta el Diagrama Termopluviométrico de Natal basado en las Normales Climatológicas 1961-1990 del observatorios de Natal (INMET), donde las temperaturas máximas oscilan entre $27,7 \circ \mathrm{C} \mathrm{C}$ y 30,5 으 y las temperaturas mínimas entre $20,7^{\circ} \mathrm{C}$ y 24 으, ya las precipitaciones con total anual de $1.617,4 \mathrm{~mm}$ donde más de $70 \%$ concentrase en el otoño e invierno. 




Figura 2 - Diagrama Termopluviométrico de Natal, Normales Climatológicas 1961-1990. Fuente: INMET.

El régimen de vientos predominantes para la ciudad está representado en la grafica y en sobre posición en el mapa de la ciudad (Figura 3), donde en más de $90 \%$ de los casos la dirección predominante es de sureste y con la velocidad del viento oscilando en un rango entre $3,6 \mathrm{~m} / \mathrm{s}$ y $5,7 \mathrm{~m} / \mathrm{s}$. Así Natal presenta un régimen de vientos de grande variación estacional, con vientos más intensos en los meses entre agosto a noviembre y más blandos entre los meses de febrero a mayo (Cosern, 2003).

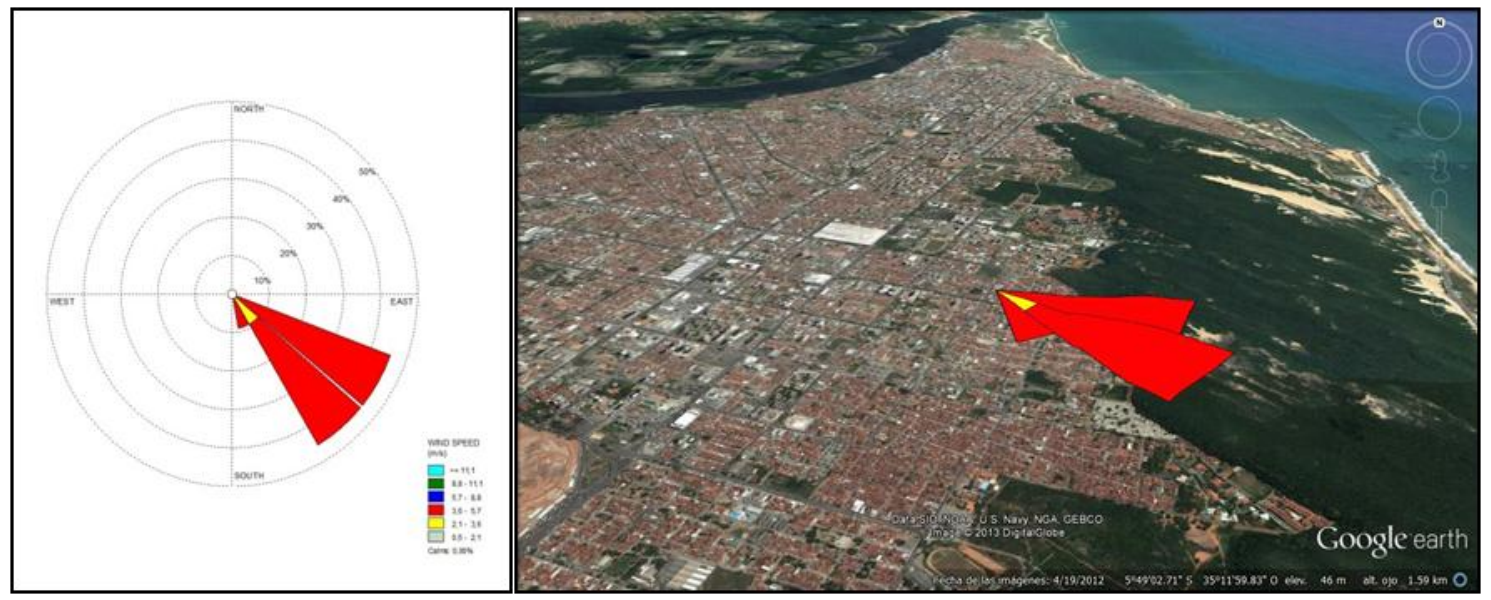

Figura 3 Dirección predominante de los vientos en la ciudad de Natal. Fuente: INMET.

Natal está situada en una zona costera (Atlántico Sur), con la presencia de áreas distintas, donde la topografía es formada por las llanuras del estuario del rio Potengi y con suelo argiloso y la presencia de la vegetación de manglar y la mayor parte de la ciudad está formado por el relieve de dunas fijas y movibles de arena, la primera recubierta con una vegetación de floresta 
de restinga y el relieve ondulado de colinas con la presencia de una vegetación florestal tropical conocida como floresta Atlántica (Nunes, 2009; Soares, 2004; Cestaro, 2002).



Figura 4 - Mapa del uso del suelo urbano de la ciudad de Natal, Brasil. Fuente: IDEMA/RN y SEMURB/Ayuntamiento de Natal.

La Figura 4 muestra el mapa de uso del suelo urbano de Natal, como se ve, el área urbana de la ciudad de Natal ocupa casi la totalidad de su área territorial como muestra en el color gris. Debido a eso, su crecimiento está acarreando daños ambientales principalmente en relación a la ocupación desordenada del suelo municipal y muy a menudo, con el "boom" inmobiliario de la construcción de edificios y viviendas (Araújo, 2007). Esto por su vez genera la preocupación con problemas climáticos urbanos fato este destacado en este estudio.

\section{MATERIALES Y MÉTODOS}

Muchos métodos se utilizan para estudiar el clima urbano y la formación de las islas térmicas, que incluyen las análisis de las series largas de temperaturas, los recorridos de campo, 
el uso de estaciones meteorológicas automáticas móviles y las imágenes de satélites (Fernández, 1996).

Fialho (2012) destaca que las innovaciones tecnológicas tales como, registradores continuos de la temperatura del aire y humedad, globos meteorológicos y las imágenes satelitales, proporcionan nuevas observaciones, lecturas y correlaciones aún combinadas entre la morfología urbana y los parámetros climáticos, permiten nuevas posibilidades de identificar las islas de calor urbanas.

Olcina y Cantos (2006) resaltan que los estudios del clima urbano se realizan mediante la comparación de datos térmicos entre la ciudad y su entorno próximo. Para obtener los datos de temperatura, humedad y vientos, señalan el uso de las técnicas de los recorridos por distintos sectores de la ciudad los transectos, las estaciones semovientes en puntos representativos, el empleo de globos sonda o globos cautivos, helicópteros, aviones o de satélites.

La metodología de estudio del clima urbano implica obligatoriamente en observaciones fijas permanentes, a demás de las observaciones móviles y episódicas y el sensoriamente remoto para subsidiar los estudios (Monteiro, 2003).

En este trabajo utilizamos las series largas de dos observatorios meteorológicos convencionales, uno ubicado en la ciudad de Natal 5055"S, 35012"W y altitud $48 \mathrm{~m}$ y el otro con una distancia de aproximadamente $10 \mathrm{~km}$ ubicado en el Aeropuerto Internacional Augusto Severo 554"S, 35015"W altitud de 51m localizado en la región metropolitana en el municipio vecino de Parnamirim, en la región metropolitana de Natal. El primero de los datos fueron fornecidos por el Instituto Nacional de Meteorología (INMET) y el segundo por el Instituto de Controle del Espacio Aéreo (ICEA).

El estudio utilizará a los análisis de las series largas de temperatura del aire para caracterizar la islas de calor de la atmosfera urbana (ICU) y su opuesto la isla de frescor de la atmosfera urbana (IFU). Para eso, se hará mediante la comparación de datos de temperaturas entre la ciudad y su entorno próximo o de misma altitud y latitud. Hay que destacar que, las diferencias de temperaturas entre el área urbana y su entorno rural tanto puede ser positiva formando una isla de calor como puede ser negativa formando una isla de frescor (Gil y Olcina, 2006). Oke (1987) define la isla de calor urbana como la diferencia de la intensidad entre los valores de la temperatura de la área urbana y la rural,

$\Delta \mathrm{t}_{\mathrm{u}-\mathrm{r}}$

Los datos estadísticos abarcaron las series largas de dados de la temperatura del aire máximas y mínimas en un periodo de 15 años seguidos, de 1995 hasta 2010 pero, con la excepción de año del 1998. Con estos datos estadísticos primarios se han utilizado las técnicas de tratamiento estadístico por lo tanto, la base de datos fue tratada y sumariada en muestreo con las medidas de posición y dispersión entre las variables. Los análisis estadísticos fueron hechos a través de los programas Excel 2007 y PSPP, como también en esa etapa fueron hechos los mapas de localización en el programa de Sistema de Información Geográfica ArcGis desktop 9.3.

Con base en las informaciones de las series largas fue posible clasificar las características, la frecuencia y intensidad de la isla de calor urbana (ICU) y de la isla de frescor urbana (IFU) para 
la ciudad de Natal. Hay que añadir que la ICU puede ser clasificada en: Débil cuando las

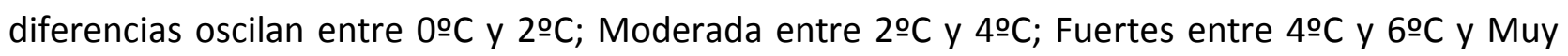
Fuerte cuando las diferencias son superiores a $6 \div \mathrm{C}$ (Fernández, 1996). Por fin fueron hechas las interpretaciones de los datos y las consideración finales y conclusión del estudio.

\section{RESULTADOS Y DISCUSIÓN}

Las oscilaciones térmicas en la atmósfera de Natal fueron evaluados entre los datos de la temperatura del aire a través de las series largas de las dos estaciones meteorológicas convencionales padrones que son, la estación meteorológica de Natal y la estación meteorológica del Aeropuerto Internacional Augusto Severo ubicado en el municipio de Parnamirim localizado en altitud y latitud muy similares a Natal.

Para eso, fueron integrados y seleccionados los datos de las temperaturas en ambos sitios y analizadas, la intensidad, la frecuencia y las variaciones temporales y la caracterización de la isla de calor de la atmósfera urbana (ICU) y de la isla de frescor de la atmósfera urbana (IFU) en la ciudad de Natal.

Para tener una primera visión general del comportamiento de las oscilaciones de las temperaturas del aire en Natal fueran integrados los promedios de las variables temperaturas máximas y mínimas en los dos puntos de análisis que son la Estación Meteorológica de Natal y el Aeropuerto en el periodo entre 1995 hasta 2010 en el Cuadro 1.

Así, en el Cuadro 1 se muestra los promedios de las temperaturas máximas quinquenales de Natal con 29,8 ㅇ C resta 30,4 ㅇ C del aeropuerto con una diferencia de $0,6 \circ \mathrm{C}$ a favor del aeropuerto. En cuanto a los promedios de las mínimas Natal presenta 22,8 ㅇ $\mathrm{C}$ y el aeropuerto de 22,3 ㄷ C con una diferencia de 0,5 ㄷ a favor de Natal. Por lo tanto para las temperaturas mínimas, la ciudad de Natal ya está como mínimo en medio grado más caliente que su entorno.

Cuadro 1 Temperaturas anuales de Natal y Aeropuerto, período de 1995-2010

\begin{tabular}{c|c|c}
\hline Estaciones Meteorológicas Convencionales & Máxima & Mínima \\
\hline Natal INMET & 29,8 & 22,8 \\
\hline Aeropuerto & 30,4 & 22,3 \\
\hline Resta Natal INMET y Aeropuerto & $-\mathbf{0 , 6}$ & $\mathbf{0 , 5}$ \\
\hline
\end{tabular}

En las Figuras 5 y 6 está representado el régimen térmico anual de los correspondientes promedios quinquenales para las temperaturas máximas y mínimas de Natal y el aeropuerto.

Así, los promedios de las temperaturas máximas mensuales de Natal oscilan entre 28,5 으 y $30,8^{\circ} \mathrm{C}$ y en el aeropuerto oscilan entre $29,1^{\circ} \mathrm{C}$ y $31,3^{\circ} \mathrm{C}$ (Figura 5). Como se ve, las temperaturas máximas del aeropuerto en todo el periodo son mayores que en Natal. 
Temperatura Máxima mensual de Natal y Aeropuerto, de 1995 hasta 2010

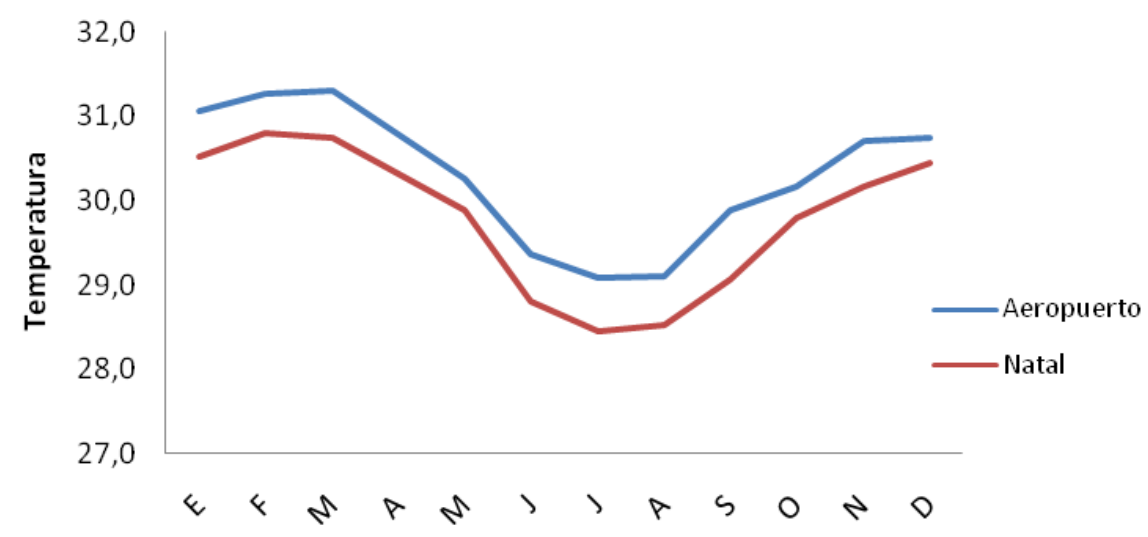

Figura 5 - El promedio mensual de las temperaturas máximas de Natal y Aeropuerto, entre 1995-2010. Fuente: INMET e ICEA

Al contrario, para las temperaturas mínimas (Figura 6) en la ciudad de Natal las temperaturas oscilan entre $20,5 \circ \mathrm{C}$ y $24,4{ }^{\circ} \mathrm{C}$ y en el aeropuerto entre 20,8 으 y $23,5 \circ \mathrm{C}$. Pero, hay una pequeña oscilación de las curvas con descenso estacional normal en los meses de julio y agosto, donde la ciudad presenta temperaturas más bajas que el aeropuerto y un ascenso normal en los demás diez meses del año.

Temperatura Mínima mensual de Natal y Aeropuerto, de 1995 hasta 2010

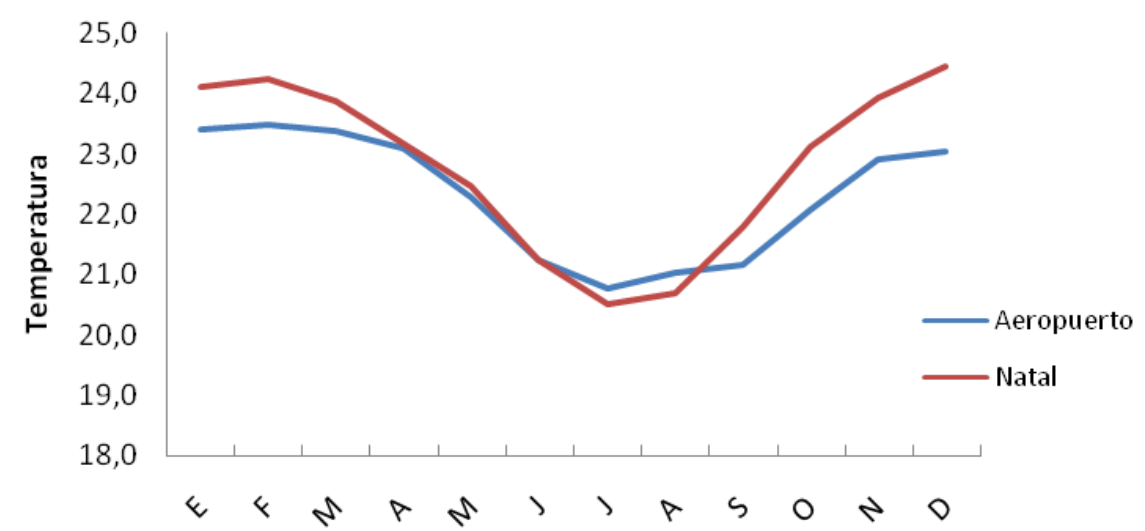

Figura 6 - El promedio mensual de las temperaturas mínimas de Natal y Aeropuerto, entre 1995-2010. Fuente: INMET e ICEA.

Conocidos los episodios anteriores de los promedios quinquenales, ahora para comprender mejor las oscilaciones térmicas de la ciudad y las presencias de las ICU y de la IFU en Natal se considerará a partir de los valores más frecuentes de los datos quinquenales del periodo en los cuatro histogramas de frecuencias entre las distintas temperaturas máximas y mínimas de Natal (Figuras 7 y 8 ) y del aeropuerto (Figuras 9 y 10).

En la Figura 7 está representado el histograma para las temperaturas máximas en Natal. Como se ve, los datos se distribuyen en una concentración de valores asimétricos positivos con 
una mayor concentración de valores entre los $28{ }^{\circ} \mathrm{C}$ y $30,5 \circ \mathrm{C}$ y con extremo más alto de 30 ㄷ․ Para las temperaturas mínimas (Figura 8) hay una distribución de valores simétricas que forman una curva ascendente con mayor con concentración de valores entre los $20,3^{\circ} \mathrm{C}$ y $24,3^{\circ} \mathrm{C}$ y con pico en $22,3 \circ \mathrm{C}$.

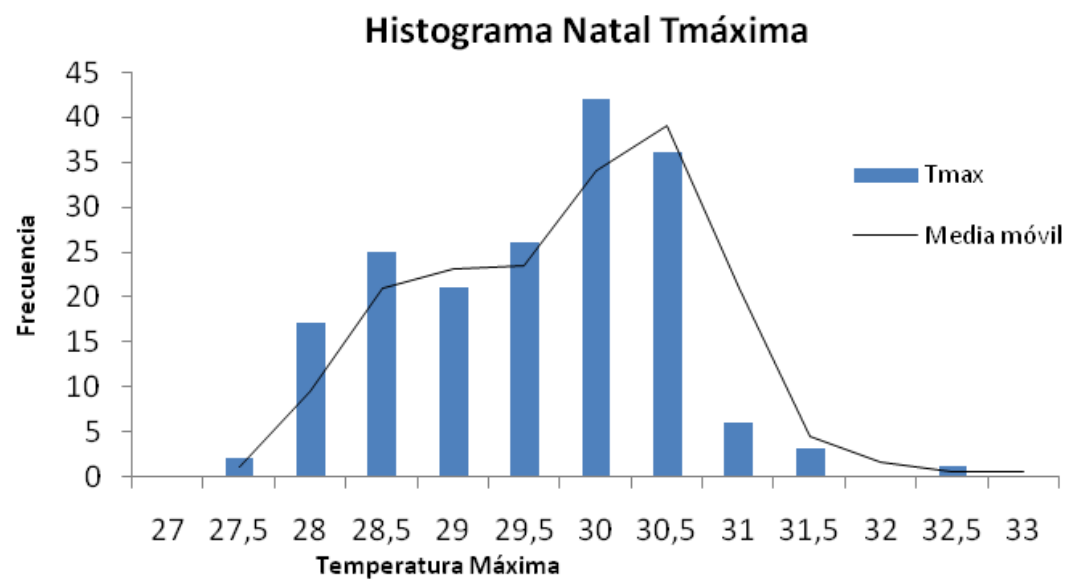

Figura 7 Histograma de frecuencia de las temperaturas máximas mensuales de Natal, entre 1995-2010. Fuente: INMET E ICEA

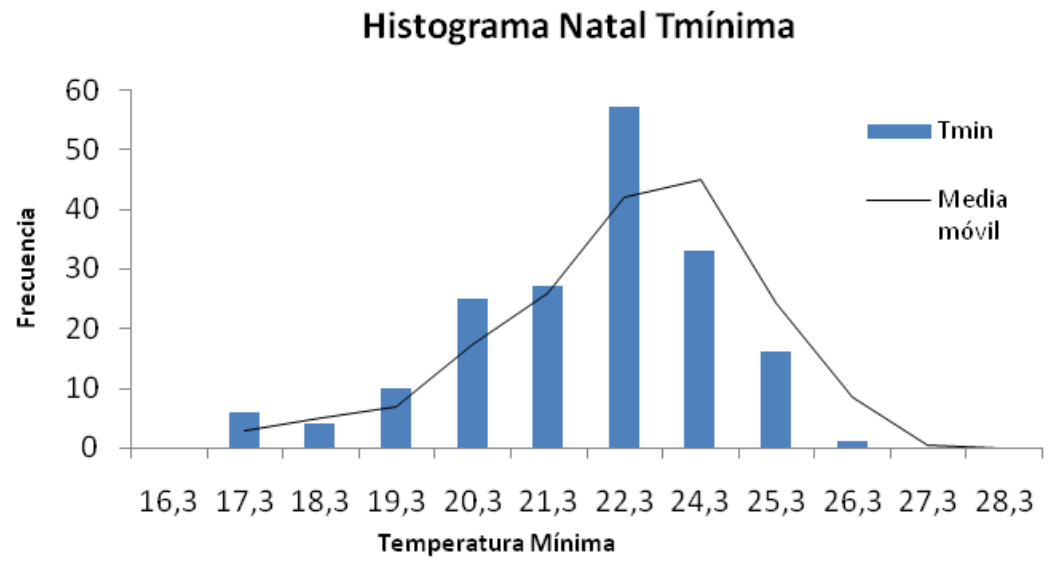

Figura 8 Histograma de frecuencia de las temperaturas mínimas mensuales de Natal, entre 1995-2010. Fuente: INMET E ICEA

Para el aeropuerto las temperaturas máximas (Figuras 9) se distribuyen en una concentración de valores claramente simétricos oscilando a partir del pico de 31 으 y con una evidente mayor concentración de valores entre los $30^{\circ} \mathrm{C}$ y $32^{\circ} \mathrm{C}$. Cuanto a las temperaturas mínimas (Figura 10) hay una distribución de valores entre los 20 ㅇ C hasta los 24,5 ㅇ C donde forman una curva muy abierta a partir del extremo más alto de 23 으. 


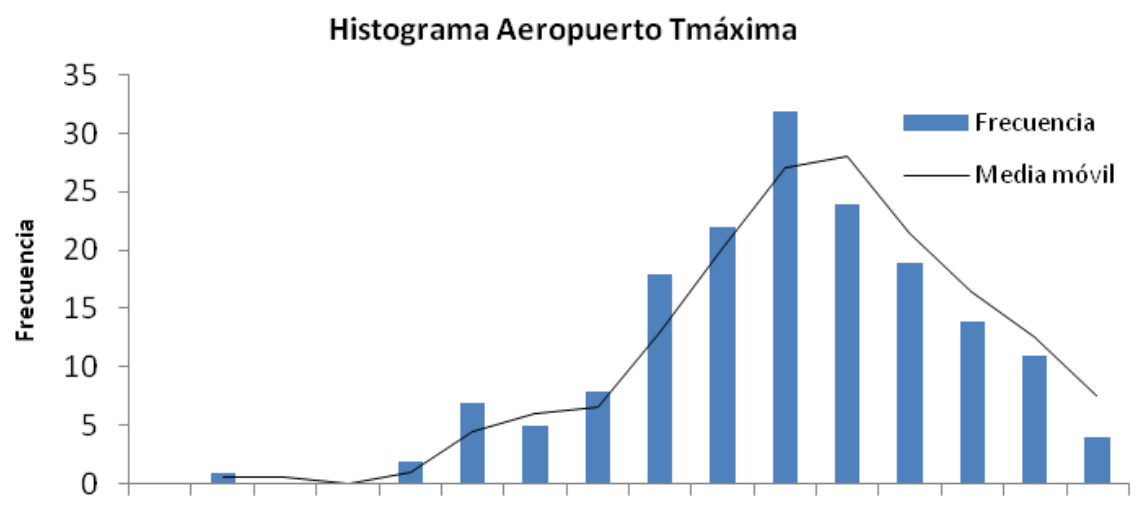

$26 \quad 26,52727,528 \quad 28,52929,530 \quad 30,531 \quad 31,532 \quad 32,5 \quad 33 \quad 33,5$ Temperatura Máximas

Figura 9 Histograma de frecuencia de las temperaturas máximas mensuales del Aeropuerto de Natal, entre 19952010. Fuente: INMET e ICEA

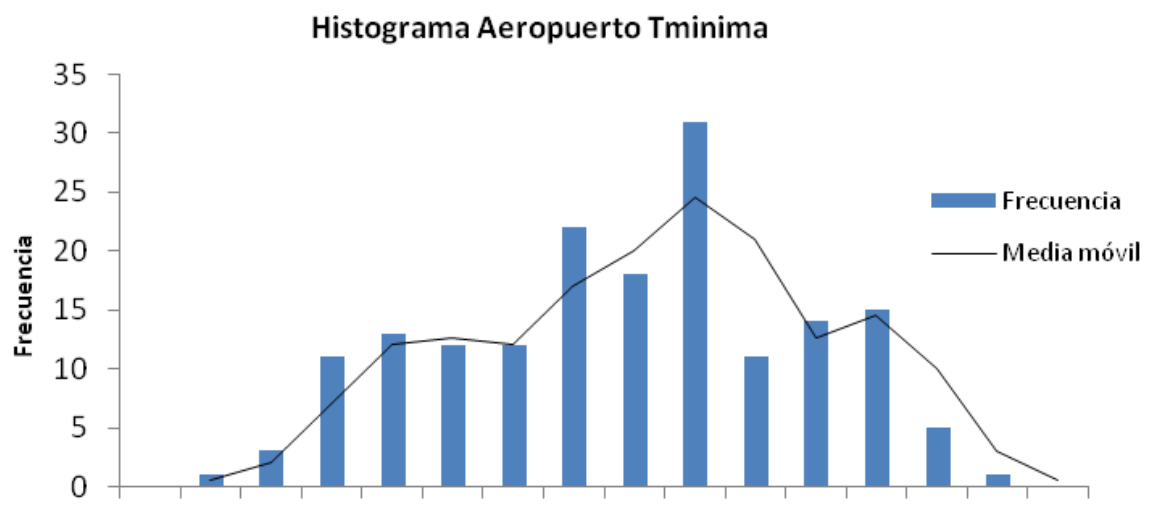

18,5 19 19,5 $2020,521 \quad 21,522 \quad 22,523 \quad 23,524 \quad 24,525 \quad 25,526$ Temperatura Minima

Figura 10 Histograma de frecuencia de las temperaturas mínimas mensuales del Aeropuerto de Natal, entre 19952010. Fuente: INMET e ICEA

\subsection{EL RITMO DE LAS ICU Y IFU}

Partiendo de las temperaturas de Natal y del aeropuerto anteriormente presentadas se esquematizan las diferencias entre las distintas temperaturas máximas y mínimas en ambos lugares para poderse determinar la intensidad y estacionalidad de las islas de calor y frescor de la atmósfera urbana (ICU y IFU) en la ciudad de Natal.

En el Cuadro 3 están representados los promedios de las diferencias de las temperaturas máximas quinquenales entre Natal (minuendo) y aeropuerto (sustraendo), como puede verse, el aeropuerto presenta 0,5 o $\mathrm{C}$ mas caliente que Natal y esos valores mayores se repiten para todos los meses. Mientras que los valores medios de las diferencias de las temperaturas mínimas Natal esta $0,6 \circ \mathrm{C}$ más caliente que el aeropuerto pero, solamente en el mes de julio Natal está 0,1 으 más baja que el aeropuerto. 
Cuadro 2 - Valores medios de las diferencias de las temperaturas máximas y mínimas entre Natal y el aeropuerto, período de 1995-2010.

\begin{tabular}{lcc}
\hline \multicolumn{1}{c}{ Mes } & Máximas & Mínimas \\
\hline Enero & $-0,5$ & 1,0 \\
\hline Febrero & $-0,5$ & 1,0 \\
\hline Marzo & $-0,5$ & 0,8 \\
\hline Abril & $-0,4$ & 0,6 \\
\hline Mayo & $-0,4$ & 0,3 \\
\hline Junio & $-0,5$ & 0,2 \\
\hline Julio & $-0,6$ & $-0,1$ \\
\hline Agosto & $-0,6$ & 0,0 \\
\hline Septiembre & $-0,8$ & 0,6 \\
\hline Octubre & $-0,6$ & 1,0 \\
\hline Noviembre & $-0,6$ & 0,9 \\
\hline Diciembre & $-0,1$ & 1,4 \\
\hline Año & $-0,5$ & 0,6 \\
\hline & Fuente: INMET e ICEA
\end{tabular}

Así para mejor caracterizar la ICU y la IFU en Natal aplicase las distintas diferencias quinquenales de los promedios de las temperaturas máximas y mínimas para una situación anual en la Figuras 10.

Para las temperaturas máximas (Figura 11) los valores se distribuyen de manera negativa y relativamente homogénea en casi todo el año a favor del aeropuerto, con la excepción de la baja en el mes de diciembre y de una oscilación mayor en el mes de septiembre. Así para las máximas el aeropuerto es mayoritariamente más caliente en todo el año que la ciudad de Natal.

Diferencias entre las temperaturas máximas y minimas entre Natal y Aeropuerto, entre 1995-2010

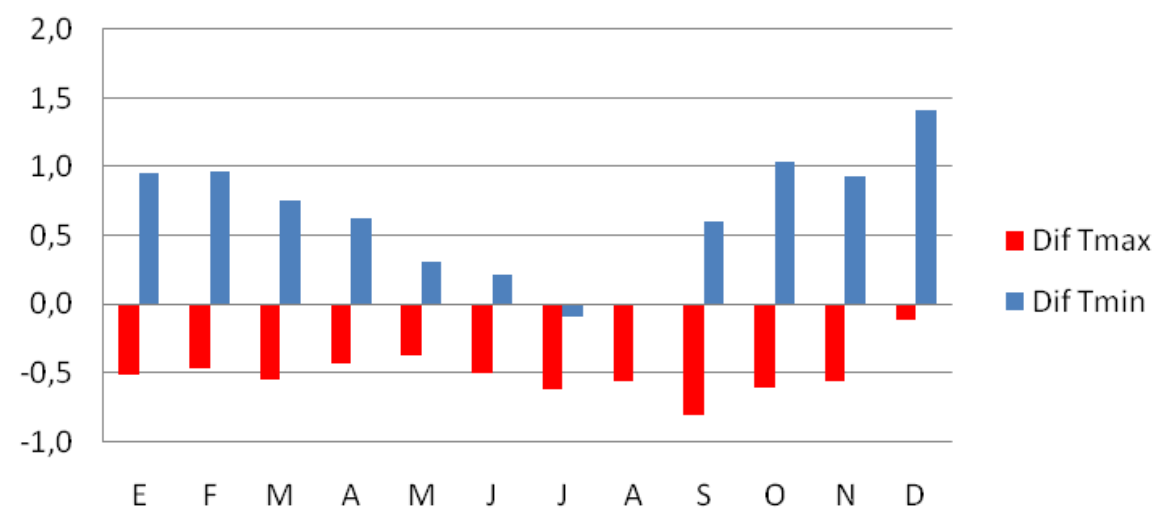

Figura 11 - Diferencias entre las temperaturas máximas y mínimas entre Natal y aeropuerto, entre 1995-2010. Fuente: INMET e ICEA 
Para las temperaturas mínimas (Figura 11) está representado un descenso gradual de las diferencias de febrero hasta en mínimo en julio en seguida un ascenso gradual hasta diciembre. Así es, las diferencias tienen valores más grandes en los meses de otoño y verano con un aplastamiento de las barras en los meses de julio y agosto, descenso este, llegando hasta ser negativo en el mes de julio mes de invierno. Esta contracción de las diferencias de las temperaturas revela que el periodo lluvioso normal entre mayo y agosto, muy a menudo, es un factor que ameniza las temperaturas en ambos lugares.

En consecuencia está visión de conjunto (Figura 11) revela que la ciudad está más caliente que su entorno en las mínimas y más fresco en las máximas. Donde, el ascenso de las diferencias entre las mínimas entre los meses de octubre hasta febrero con pico en diciembre, visualiza la formación de una ICU en el verano en la ciudad de Natal.

El siguiente paso es determinar cuáles son las frecuencias del reparto de las diferencias de las temperaturas máximas y mínimas. Ello es posible con la esquematización en los histogramas de las Figuras 12 y 13. En ellos están representados las frecuencias de las diferencias entre las temperaturas máximas y mínimas entre Natal y el aeropuerto.

La Figura 12 está representando las frecuencias de las diferencias entre las temperaturas máximas entre Natal y el aeropuerto. Lo que se observa es que hay una distribución muy repartida entre los valores, mientras los valores negativos son predominantes en más de $62 \%$ de las diferencias. Importante señalar que de estos valores $38 \%$ son arriba de los 0,1 grados y de estos hay $5 \%$ arriba de del 1 grado. A pesar de la predominancia de los valores negativos y que esto representa la presencia de una IFU en Natal, sin embargo, hay una tendencia ya marcada de una inversión, cuyas diferencias de temperaturas se acercan a los valores positivos. La situación que nos muestra es que para las temperaturas máximas, en $62 \%$ de los casos hay la presencia de una IFU en Natal y en 38\% de los casos ya presenta la formación de ICU en Natal.

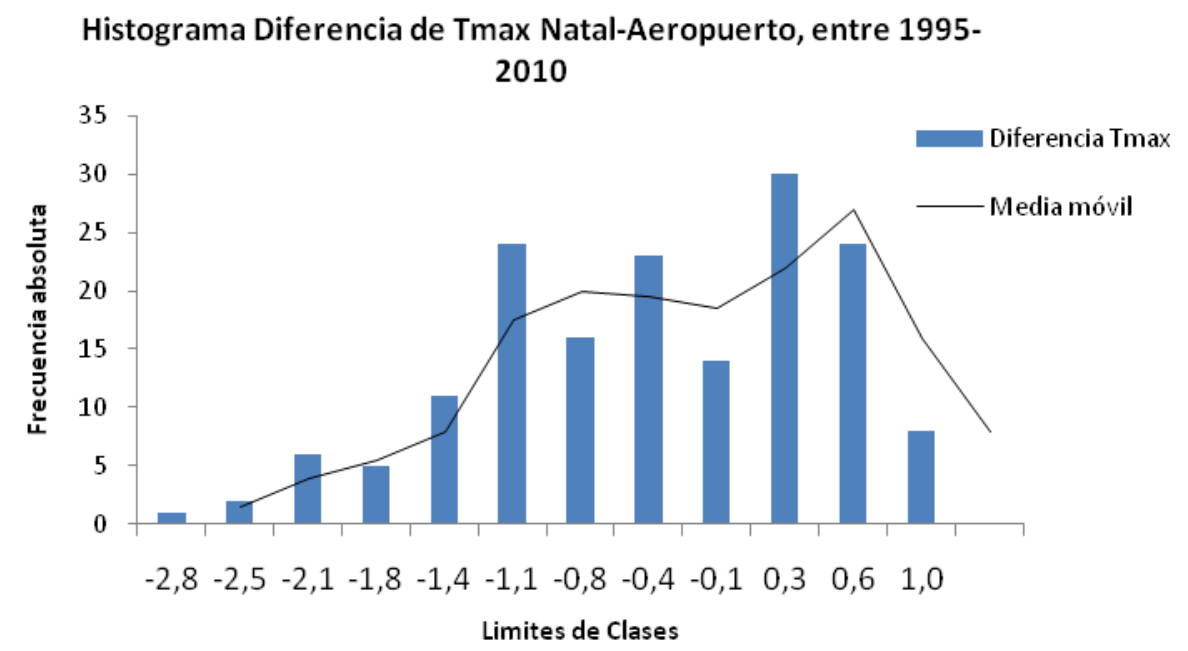

Figura 12 - Frecuencia de las diferencias entre las temperaturas máximas entre Natal y aeropuerto, entre 19952010. Fuente: INMET y ICEA Fuente: INMET e ICEA.

La Figura 13está representanda las frecuencias de las diferencias entre las temperaturas mínimas entre Natal y el aeropuerto. En ella vemos que, hay una distribución repartida con un pico en los 1,7 grados y descenso brusco a la derecha y lento a la izquierda. El hecho distintivo de 
esta situación es que en $68 \%$ de los valores de las frecuencias de las mínimas están arriba de 0.5 grados, además de $18 \%$ de los valores de las frecuencias de las mínimas están arriba de los preocupantes 2 grados. Esto viene a comprobar que para las temperaturas mínimas, en $68 \%$ de los casos hay la presencia de una ICU en Natal y en 32\% de los casos ya presenta la formación de IFU en Natal. Está muy evidente la formación de ICU en Natal.

Estos hechos nos muestran en ambos los casos hay la presencia de una ICU Débil en Natal. Muy acentuada en los $68 \%$ para las temperaturas mínimas y se expande en los $38 \%$ para las temperaturas máximas.

Histograma Diferencia de Tmin Natal-Aeropuerto, entre 1995-

2010

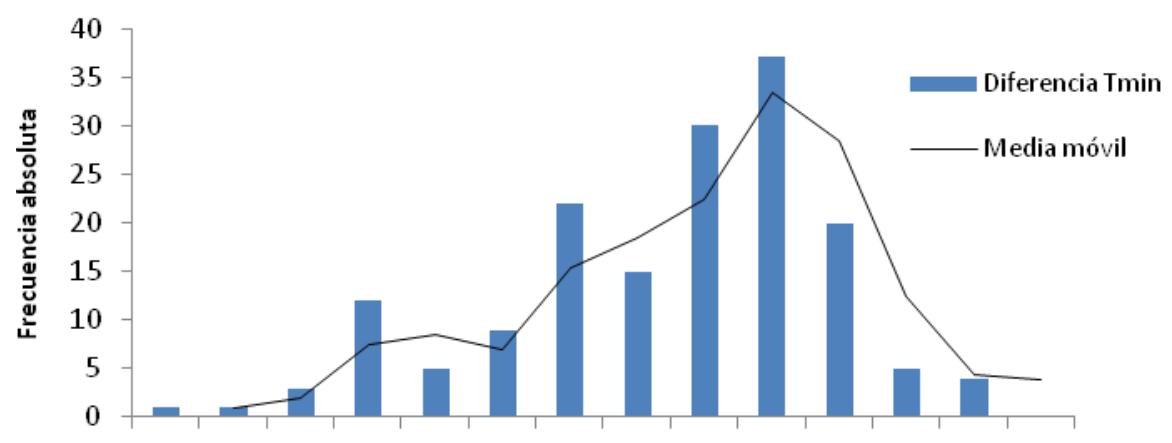

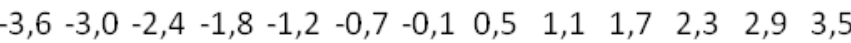

Limite de Clase

Figura 13 - Frecuencia de las diferencias entre las temperaturas mínimas entre Natal y aeropuerto, entre 19952010. Fuente: INMET y ICEA Fuente: INMET e ICEA

De todo lo expuesto hasta ahora se hace necesario determinar ¿cuáles son las probabilidades de que se produzca ICU y IFU y su intensidad en Natal? Para despejar esta cuestión el análisis a través de los cálculos de los valores de los Cuantiles y Percentiles son útiles cuando se necesita establecer la posición relativa de valores en grupo de datos (Mafokozi, 2009).

Así en el cuadro 3 está el resultado quinquenal (1995-2010) por meses de las diferencias entre las temperaturas máximas (que representan la IFU) entre Natal menos el aeropuerto.

De los resultados obtenidos (cuadro 3 ) los que más se distingue es el nonagésimo percentil donde todos los valores se superan a $\operatorname{los} 0,5 \circ \mathrm{C}$, lo que significa que, de todo el quinquenio, como mínimo en $10 \%$ de los $100 \%$ de los días Natal presenta 0,5 ㄷ por encima del aeropuerto que es una ICU Débil. Los valores de la IFU están representados en el quincuagésimo percentil donde todos los valores son negativos, por lo tanto, las diferencias para las temperaturas máximas Natal presenta temperaturas máximas menores que el aeropuerto.

Cuadro 3 - Valores de los Cuantiles y Percentiles por meses de las diferencias entre las temperaturas máximas entre Natal menos el aeropuerto, período de 1995-2010.

\begin{tabular}{l|c|c|c|c|c|c|c|c|c|c|c|c|c|c|c}
\hline \multicolumn{1}{c|}{ Mes } & Mínimo & P10 & Q1 & P25 & Q2 & Mediana & P50 & Promedio & Moda & Q3 & P75 & P90 & P99 & Q4 & Máximo \\
\hline Enero & $-6,7$ & $-2,1$ & $-1,4$ & $-1,2$ & $-0,4$ & $-0,4$ & $-0,4$ & $-0,5$ & 0,0 & 0,2 & 0,2 & $\mathbf{0 , 8}$ & 2,3 & 6,2 & 6,2 \\
\hline Febrero & $-4,2$ & $-2,0$ & $-1,3$ & $-1,3$ & $-0,3$ & $-0,3$ & $-0,3$ & $-0,5$ & 0,0 & 0,3 & 0,3 & $\mathbf{0 , 8}$ & 2,3 & 3,1 & 3,1 \\
\hline Marzo & $-3,8$ & $-2,0$ & $-1,2$ & $-1,2$ & $-0,3$ & $-0,3$ & $-0,3$ & $-0,4$ & 0,0 & 0,2 & 0,2 & $\mathbf{0 , 9}$ & 2,2 & 4,0 & 4,0 \\
\hline
\end{tabular}




\begin{tabular}{l|c|c|c|c|c|c|c|c|c|c|c|c|c|c|c}
\hline Abril & $-6,4$ & $-2,2$ & $-1,4$ & $-1,4$ & $-0,4$ & $-0,4$ & $-0,4$ & $-0,5$ & 0,0 & 0,3 & 0,3 & $\mathbf{1 , 0}$ & 2,0 & 3,4 & 3,4 \\
\hline Mayo & $-6,7$ & $-2,0$ & $-1,4$ & $-1,4$ & $-0,4$ & $-0,4$ & $-0,4$ & $-0,4$ & 0,2 & 0,4 & 0,4 & $\mathbf{1 , 2}$ & 2,8 & 5,7 & 5,7 \\
\hline Junio & $-3,0$ & $-1,9$ & $-1,3$ & $-1,3$ & $-0,4$ & $-0,4$ & $-0,4$ & $-0,4$ & $-0,2$ & 0,4 & 0,4 & $\mathbf{1 , 2}$ & 2,9 & 6,2 & 6,2 \\
\hline Julio & $-5,3$ & $-2,2$ & $-1,3$ & $-1,3$ & $-0,6$ & $-0,6$ & $-0,6$ & $-0,5$ & $-0,8$ & 0,3 & 0,3 & $\mathbf{1 , 0}$ & 4,7 & 5,5 & 5,5 \\
\hline Agosto & $-5,4$ & $-2,4$ & $-1,4$ & $-1,4$ & $-0,5$ & $-0,5$ & $-0,5$ & $-0,6$ & 0,0 & 0,2 & 0,2 & $\mathbf{0 , 8}$ & 2,9 & 5,3 & 5,3 \\
\hline Septiembre & $-4,0$ & $-2,0$ & $-1,4$ & $-1,4$ & $-0,6$ & $-0,6$ & $-0,6$ & $-0,6$ & $-1,4$ & 0,2 & 0,2 & $\mathbf{0 , 8}$ & 2,1 & 3,8 & 3,8 \\
\hline Octubre & $-4,8$ & $-2,3$ & $-1,5$ & $-1,5$ & $-0,7$ & $-0,7$ & $-0,7$ & $-0,8$ & $-0,8$ & 0,0 & 0,0 & $\mathbf{0 , 5}$ & 1,3 & 2,6 & 2,6 \\
\hline Noviembre & $-3,6$ & $-2,0$ & $-1,3$ & $-1,3$ & $-0,5$ & $-0,5$ & $-0,5$ & $-0,6$ & $-0,2$ & 0,1 & 0,1 & $\mathbf{0 , 7}$ & 1,5 & 2,3 & 2,3 \\
\hline Diciembre & $-3,2$ & $-2,0$ & $-1,3$ & $-1,3$ & $-0,4$ & $-0,4$ & $-0,4$ & $-0,5$ & $-0,6$ & 0,2 & 0,2 & $\mathbf{0 , 8}$ & 1,8 & 1,9 & 1,9 \\
\hline Año & $-3,4$ & $-2,3$ & $-1,4$ & $-1,4$ & $-0,4$ & $-0,4$ & $-0,4$ & $-0,6$ & 0,0 & 0,4 & 0,4 & $\mathbf{0 , 8}$ & 1,6 & 3,0 & 3,0 \\
\hline
\end{tabular}

Así en el cuadro 4 está el resultado quinquenal (1995-2010) por meses de las diferencias entre las temperaturas mínimas que representan la IFU entre Natal menos el aeropuerto.

Se puede resaltar que, los valores del tercer cuartil muestran que en los meses de enero, febrero, marzo, septiembre, octubre, noviembre y diciembre en los $25 \%$ de los $100 \%$ de los días Natal presenta más de $1,0 \circ \mathrm{C}$ de temperatura mayor que el aeropuerto. Esto significa que en todo el quinquenio un cuarto de todos los días Natal presenta una ICU débil.

También en este cuadro 4, de los resultados obtenidos se observa que el nonagésimo percentil se superan en los $2^{\circ} \mathrm{C}$ en los meses de febrero, octubre, noviembre y diciembre, además de los 1,9 ㅇ $\mathrm{C}$ en enero y septiembre, lo que significa que, de todo el quinquenio, como mínimo en $10 \%$ de los $100 \%$ de los días Natal está 2 ㄷ C por encima de su entorno que es una muy preocupante ICU Moderada.

Cuadro 4 - Valores de los Cuantiles y Percentiles por meses de las diferencias entre las temperaturas mínimas entre Natal menos el aeropuerto, período de 1995-2010.

\begin{tabular}{l|c|c|c|c|c|c|c|c|c|c|c|c|c|c|c}
\hline \multicolumn{1}{c|}{ Mes } & Mínimo & P10 & Q1 & P25 & Q2 & Mediana & P50 & Promedio & Moda & Q3 & P75 & P90 & P99 & Q4 & Máximo \\
\hline Enero & $-6,1$ & $-3,2$ & $-1,7$ & $-1,7$ & 0,0 & 0,0 & 0,0 & $-0,3$ & $-0,9$ & $\mathbf{1 , 2}$ & $\mathbf{1 , 2}$ & 1,9 & 3,0 & 3,8 & 3,8 \\
\hline Febrero & $-5,9$ & $-3,2$ & $-1,1$ & $-1,1$ & 0,2 & 0,2 & 0,2 & $-0,1$ & 0,1 & $\mathbf{1 , 2}$ & $\mathbf{1 , 2}$ & $\mathbf{2 , 1}$ & 3,1 & 4,0 & 4,0 \\
\hline Marzo & $-6,3$ & $-3,3$ & $-1,3$ & $-1,3$ & 0,0 & 0,0 & 0,0 & $-0,4$ & 0,3 & $\mathbf{1 , 0}$ & $\mathbf{1 , 0}$ & 1,8 & 3,3 & 4,2 & 4,2 \\
\hline Abril & $-5,9$ & $-3,4$ & $-1,5$ & $-1,5$ & $-0,2$ & $-0,2$ & $-0,2$ & $-0,6$ & $-0,1$ & 0,6 & 0,6 & 1,2 & 2,2 & 4,2 & 4,2 \\
\hline Mayo & $-6,4$ & $-3,9$ & $-1,9$ & $-1,9$ & $-0,5$ & $-0,5$ & $-0,5$ & $-0,8$ & 1,0 & 0,6 & 0,6 & 1,2 & 2,4 & 3,6 & 3,6 \\
\hline Junio & $-6,3$ & $-3,6$ & $-2,1$ & $-2,1$ & $-0,9$ & $-0,9$ & $-0,9$ & $-1,1$ & $-0,3$ & 0,2 & 0,2 & 0,8 & 2,0 & 3,1 & 3,1 \\
\hline Julio & $-5,3$ & $-3,9$ & $-2,7$ & $-2,7$ & $-1,1$ & $-1,1$ & $-1,1$ & $-1,3$ & $-2,9$ & 0,1 & 0,1 & 0,9 & 2,0 & 4,1 & 4,1 \\
\hline Agosto & $-6,5$ & $-3,9$ & $-2,7$ & $-2,7$ & $-0,8$ & $-0,8$ & $-0,8$ & $-1,1$ & 0,9 & 0,6 & 0,6 & 1,3 & 2,3 & 3,4 & 3,4 \\
\hline Septiembre & $-6,3$ & $-3,2$ & $-2,1$ & $-2,1$ & $-0,5$ & $-0,5$ & $-0,5$ & $-0,6$ & $-2,9$ & $\mathbf{1 , 1}$ & $\mathbf{1 , 1}$ & 1,9 & 3,2 & 3,8 & 3,8 \\
\hline Octubre & $-7,3$ & $-3,5$ & $-1,9$ & $-1,9$ & 0,0 & 0,0 & 0,0 & $-0,4$ & 0,9 & $\mathbf{1 , 3}$ & $\mathbf{1 , 3}$ & $\mathbf{2 , 2}$ & 3,2 & 4,5 & 4,5 \\
\hline Noviembre & $-5,2$ & $-3,1$ & $-2,1$ & $-2,1$ & 0,0 & 0,0 & 0,0 & $-0,3$ & 0,3 & $\mathbf{1 , 4}$ & $\mathbf{1 , 4}$ & $\mathbf{2 , 1}$ & $\mathbf{2 , 9}$ & 3,6 & 3,6 \\
\hline Diciembre & $-5,4$ & $-3,3$ & $-1,5$ & $-1,5$ & 0,4 & 0,4 & 0,4 & $-0,1$ & 0,8 & $\mathbf{1 , 5}$ & $\mathbf{1 , 5}$ & $\mathbf{2 , 3}$ & 3,4 & 3,7 & 3,7 \\
\hline Año & $-7,3$ & $-3,5$ & $-1,9$ & $-1,5$ & $-0,3$ & $-0,3$ & $-0,3$ & $-0,6$ & $-0,9$ & $\mathbf{0 , 9}$ & $\mathbf{0 , 9}$ & 1,8 & 3,0 & 4,5 & 4,5 \\
\hline
\end{tabular}

Estos datos permiten posicionar que hay una oscilación de temperaturas en la atmósfera urbana de la ciudad de Natal y también la caracterización y formación de las ICU y la IFU en la ciudad. Hay que señalar que las situaciones sinópticas predominantes (ZCIT, los Sistemas 
Frontales y los vientos Alisios del sureste) son determinantes para el clima y las oscilaciones de temperaturas en la región de Natal.

Respecto a los datos de este estudio es importante destacar que presentan las medias diarias y no horarias, por lo tanto, los resultados muestran la presencia de las ICU y IFU en días y no en horarios.

Ante los resultados, se afirma que, mismo en diferentes ritmos, la estacionalidad de la ICU es muy presente en el otoño y verano, así com una intensidad por encima de los 0,9 o $C$ en estos periodos. Para la IFU en Natal, los datos presentan que en todo el año, en los meses de abril, mayo, junio y julio es un poco más influyente con temperaturas de más de $1,0 \circ \mathrm{C}$ de diferencia entre Natal y aeropuerto.

Siendo Natal una ciudad de clima tropical en la cual el calentamiento urbano, por si solo, ya es algo muy preocupante para la población de la ciudad (NIMER, 1979). Los resultados obtenidos muestran que esta ciudad tiene la presencia de una ICU Débil como mínimo en $25 \%$ de los días y con una temperatura del aire 0,9 ㅇ C por encima del aeropuerto o mejor su entorno. $Y$ una ICU Moderada presente en $10 \%$ de los días con valores encima de $2{ }^{\circ} \mathrm{C}$ en la ciudad de Natal.

Todo ello determinará diferencias sustanciales para la caracterización de la isla de calor y la isla de frescor de la atmosfera urbana de Natal, pero de hecho, es en la ICU donde las respectivas diferencias entre las correspondientes temperaturas mínimas se presentan los valores más destacadamente preocupantes y mayores que para la ciudad de Natal.

\section{CONCLUSIONES}

La caracterización de una ICU y IFU a través de las series largas de la temperatura de aire es unos de los métodos clásicos en climatología (Fernández García, 1996). Por ello, en la ciudad de Natal se permite este tipo de método debido a los datos de la estación meteorológica instalada en su área urbana y otra a $10 \mathrm{~km}$ de distancia en el aeropuerto un sitio que a pesar de la presencia de la ciudad de Parnamirin aún tiene características rurales.

La comparación de los datos de la temperatura del aire entre las dos estaciones meteorológicas a través del análisis estadístico en el quinquenio 1995-2010 fue hecha con un total de más de 5.400 días.

Del total de los 5.440 días la ciudad de Natal presentó para las temperaturas máximas más de 1.580 días con la formación de isla de calor, eso es, las temperaturas máximas de Natal mayores que los del Aeropuerto y para la temperatura mínima fueron 2.408 días con la formación de isla de calor, eso es, las temperaturas mínimas de Natal mayores que los del Aeropuerto.

Las variables que explican las diferencias térmicas fueron una intensidad por encima de los 0,9 ㅇ muy marcada en los meses de otoño y verano para el quinquenio caracterizado como una ICU Débil. 
Un dato importante y preocupante del resultado es que, en $10 \%$ de los 5.440 días Natal tiene una temperatura del aire $2 \stackrel{\circ}{\circ}$ por encima del aeropuerto, lo cual explica la presencia de una ICU Moderada.

La presencia de una ICU en una ciudad de clima tropical como Natal es algo preocupante y requiere políticas urbanas sostenibles y con una clara visión ambiental, porque al final los risesgos son claros, provocando evidentes problemas ambientales, además de económicos y sociales.

\section{BIBLIOGRAFÍA}

AYOADE, J. O. (2003). Introdução à climatologia para os trópicos. 9 ed. Rio de Janeiro, Bertrand Brasil.

ARAÚJO DA COSTA, Ademir. (2007). A verticalização de Natal, elemento de impactos socioambientales sobre o seu desenvolvimento urbano. In Nunes, Elias (coord.). Dinâmica e Gestão do Território Potiguar. Natal, EDUFRN.

ARAÚJO, Ronaldo Rodrigues; RANGEL, Mauricio Eduardo Salgado. (2012). Crescimento urbano e variações térmicas em São Luis MA. Revista Geonorte, Edicion Especial 2, v.2, n. 5, p. 308 a 318.

ASSIS, Eleonora Sad de. (2006). Aplicações da climatologia urbana no planejamento da cidade: revisão dos estudos brasileiros. Revista de Urbanismo e Arquitetura, Vol. 7, N. 1.

BALDICERO MOLION, Luiz Carlos; OLIVEIRA BERNARDO, Sergio de. (2002). Uma revisão da dinamica das chuvas no Nordeste brasileiro. Brasil, Revista Brasileira de Meteorologia, v 17, n.1.

BARRY, Roger G.; CHORLEY, Richard J. (2003). Atmosphere, weather and climate. 8 edition. London, Routledg.

BRANDÃO, Ana Maria de Paiva Macedo. (2003). O clima urbano da cidade do Rio de Janeiro. In Mendonça, Francisco; Monteiro, Carlos Augusto de Figueiredo (orgs.). Clima urbano. São Paulo, Contexto.

CORRÊA, Roberto Lobato. (1989). O espaço urbano. São Paulo: editora Ática.

COSERN. (2003) Potencial eólico do estado do Rio Grande do Norte. Natal: Companhia Energética do Rio Grande do Norte., pp 47.

FIALHO, Edson Soares. (2012). Ilha de calor: reflexões acerca de um conceito. ACTA Geográfica, Boa Vista, Ed. Esp. Climatologia Geográfica, pp. 61-76.

GIL OLCINA, Antonio; OLCINA CANTOS, Jorge. (2006). Climatología general. 2o Edición. Barcelona, Ariel.

SOARES CUNHA, Eugenio Marcos. (2004). Evolución actual del litoral de Natal RN, Brasil y sus aplicaciones a la Gestión Integrada. Barcelona: Universitat de Barcelona, Tesis Doctoral.

CESTARO, Luiz Antonio. (2002). Fragmentos de florestas Atlânticas no Estado do Rio Grande do Norte relações estruturais floristicas e fitogeográficas. São Carlos, UFSCar, Tese de Docorado. 
Emmanuel, Rohinton. (2011). Urban climate design in the equatorial megapolis Key challenges to effective adaptive action. http://www.forumpatrimonio.com.br/print.php?articlelD=199\&modo=1

FERNÁNDEZ GARCÍA, Felipe. (1996). Manual de Climatología Aplicada, clima, medio ambiente y planificación. Madrid, Editorial Síntesis.

FIALHO, Edson Soares. (2012). Ilha de calor: reflexões acerca de um conceito. Boa Vista, ACTA Geográfica, Ed. Esp Climatología, pp. 61-76.

IBGE.

Cidades,

Natal.

http://www.ibge.gov.br/cidadesat/painel/painel.php?codmun=240810

LÓPEZ GÓMES, Antonio (coord.). (1993). El clima de las ciudades españolas. Madrid, Ediciones Cátedra.

MAFOKOZI, Joseph. (2009). Introducción a la estadistica. Madrid, Editorial CCS.

MONTEIRO, Carlos Augusto de Figueiredo; MENDONÇA, Antonio (orgs.). (2003). Clima urbano. São Paulo, Editora Contexto.

MOREIRA, Elvis Bergue Mariz; GALVÍNCIO, Josiclêda Domiciano. (2007). Espacialização das temperaturas à superfície na cidade do Recife Utilizando imagens tm Landsat 7.

MOURA, Marcelo de Oliveira; ZANELLA, Maria Elisa; SALES, Marta Celina Linhares. (2008). Ilhas térmicas na cidade de Fortaleza CE.

NATAL. (2012). Anuário Natal 2011 e 2012. Natal: PMN, SEMURB.

NIMER, Edmond. (1979). Um modelo metodológico da classificação de climas. Rio de Janeiro, IBGE, Revista Brasileira de Geografia, nr 4, ano 41, p. 59-89, out/dez.

NUNES, Elias. (2009). O meio ambiente da Grande Natal. 2 ed. Natal: Imagem Gráfica.

OLIVIER, John E. (2005). Encyclopedia of world climatology. Netherlands, Springer.

OKE, T.R. (1987). Boundary layer climates. 2th Edition. Routledge, London.

SANTOS, Milton. (1999). A Natureza do Espaço: Técnica e Tempo; Razão e Emoção. 3a edicion. São Paulo: Hucitec. 